\title{
OBJECIONES DE PRINCIPIO. RESPUESTA A ALEKSANDER PECZENIK Y LUIS PRIETO SANCHÍS
}

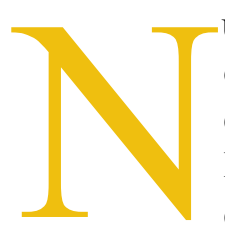

uestro trabajo sobre los principios jurídicos ha sido objeto de un buen número de críticas, la mayor parte formuladas oralmente en diversos seminarios en los que hemos tenido ocasión de discutirlo ${ }^{1}$.

En uno de estos seminarios -el II Spanish-Finnish Seminar on Legal Theory celebrado en Tampere en septiembre del año pasado- Aleksander Peczenik presentó un breve trabajo que se publica en este mismo número de Doxa y que viene a ser una crítica global de la concepción de los principios jurídicos defendida por nosotros. Otro tanto ocurre -aunque obviamente la discusión es aquí más amplia y no se circunscribe a nuestras tesis, sino que abarca las diversas concepciones de los principios jurídicos presentes en la actual discusión iusfilosófica- con el capítulo primero del libro de inminente aparición de Luis Prieto Sanchís sobre los principios en el Derecho.

Esta nota trata de responder a ambos. Vale la pena indicar de entrada que tanto Peczenik como Prieto critican los ejes centrales de nuestra contribución y que, con las naturales diferencias de acento, en sus textos podemos encontrar asimismo lo esencial de las críticas que verbalmente nos han hecho otros filósofos del Derecho. Tratar de responderles constituye, pues, un excelente bando de pruebas para nosotros.

Lo esencial de las críticas que nos dirigen Prieto y Peczenik puede -nos pareceagruparse en los dos puntos siguientes: a) nuestra defensa del entendimiento de las reglas jurídicas de mandato

${ }^{1}$ Una primera versión de este trabajo se publicó en el n. ${ }^{0} 10$ de Doxa con el título de «Sobre principios y reglas» y fue discutida en el seminario sobre problemas actuales de la teoría del Derecho en Estados Unidos y en España celebrado en el Centro de Estudios Constitucionales en junio de 1991. Una versión posterior, más amplía, con el título de «Three Approaches to Legal Principles» fue presentada en el II Finnish-Spanish Seminar on Legal Theory y se encuentra pendiente de publicación. 
como razones perentorias y nuestra consideración de que tales reglas se diferencian de los principios en sentido estricto en que aquellas configuran el caso de forma «cerrada» mientras que éstos lo hacen de forma «abierta»; b) nuestra concepción de los principios en sentido estricto como normas que exigen un cumplimiento pleno, esto es, que no admiten modalidades graduables de cumplimiento. Podría pensarse que en (a) hay al menos dos cuestiones distintas, pero nos parece que, tanto en nuestro planteamiento como en las críticas de Prieto y Peczenik, se trata más bien de dos aspectos distintos de la misma cuestión: pues el que las reglas puedan operar como razones perentorias implica, como condición necesaria, el que determinen de forma «cerrada» sus condiciones de aplicación.

a) Las reglas de mandato como razones perentorias y los principios como razones no perentorias; determinación "cerrada" o "abierta" de las condiciones de aplicación.

En Sobre principios y reglas asumimos, por un lado, la caracterización de las normas de Alchourrón y Bulygin como correlaciones entre casos genéricos (conjuntos de propiedades) y soluciones normativas y, por otro, la caracterización de Hart de las razones jurídicas autoritativas como razones perentorias e independientes del contenido. Asumimos ambas caracterizaciones circunscribiendo expresamente su alcance a las reglas jurídicas de mandato, esto es, dejando fuera otros tipos de reglas que (como las permisivas o las que confieren poderes) quedaban fuera de nuestra consideración y dejando fuera también, ya dentro de nuestro tema, a los principios, a los que tratábamos de caracterizar estructuralmente en contraposición a la caracterización ofrecida por Alchourrón y Bulygin $\mathrm{y}$, desde la perspectiva que llamábamos funcional, en contraposición a la caracterización hartiana. Nuestros críticos han captado bien el engarce entre ambas asunciones: pues, en efecto, si las reglas pueden constituir no sólo razones de primer orden para realizar la acción exigida sino también razones de segundo orden para, en los términos de Hart, «excluir o suprimir cualquier deliberación independiente por parte de su destinatario sobre los argumentos en pro y en contra de realizar la acción», ello es sólo porque configuran de forma «cerrada» sus condiciones de aplicación. Tanto Prieto como Peczenik siguen, desde perspectivas distintas, un mismo hilo argumentativo: la pretendida configuración «cerrada» de las condiciones de aplicación no es tal y, por tanto, las reglas no pueden constituir razones perentorias. 
a') Las críticas de Prieto

Empecemos con las objeciones de Prieto, que citamos in extenso para tratar de analizarlas posteriormente paso a paso. Con arreglo a cierta interpretación -escribe este autor-.

«lo que diferenciaría a reglas y principios es que a priori o antes de su aplicación podemos discernir con precisión en qué casos deberá observarse una norma, dado que el ordenamiento prevé o debe prever todas las posibles excepciones a su aplicación, mientras que los principios no disponen ni pueden disponer de una cláusula de este género. [...] En consecuencia, la diferencia residiría en que así como podemos conocer a ciencia cierta cuando se impone la solución prevista en una norma, existe un margen de duda a propósito de la solución avalada por un principio».

Entre quienes sostienen esta tesis, Prieto cita a Dworkin y a nosotros: «Aunque con importantes diferencias respecto de Dworkin, me parece que entre nosotros M. Atienda y J. Ruiz Manero siguen una exploración semejante». Cita nuestra tesis de que las reglas configuran de forma «cerrada» sus condiciones de aplicación mientras que los principios lo hacen de forma «abierta» y añade que «a este criterio de distinción estructural Atienza y Ruiz Manero superponen otro funcional», esto es, la consideración de que, mientras que las reglas constituyen razones perentorias, los principios son meramente razones de primer orden. «Si he entendido bien -prosigue- esto significa que las reglas deben contener todas las excepciones posibles a su aplicación y de ahí que, seleccionada la norma, no sea preciso ponderar ninguna otra norma o principio; los principios, en cambio, se caracterizarían por concurrir con otros principios». Esta manera de entender la distinción entre reglas y principios estaría, a juicio de Prieto, viciada de raíz por partir de «una errónea presentación de las reglas». Pues,

«en efecto, muchas veces en el razonamiento jurídico las reglas aparecen como criterios hermenéuticos y no como reglas específicas y exhaustivamente comprensivas del caso examinado, exigen también con frecuencia conjugarse con otros estándares jurídicos (principios o normas), lo que matiza o altera su alcance y el propio ámbito de aplicación y, en fin, ofrecen siempre una textura abierta o vaguedad potencial en su significado, de modo que no enuncian de un modo completo y absoluto un catálogo de posibles excepciones a su aplicación. En este sentido, la postura comentada parece mostrar excesiva confianza en la finitud lógica, ya que no 
del sistema en su conjunto, al menos del sistema de reglas». Pues «parece bastante improbable que alguien fuera capaz de catalogar por completo las posibles excepciones a la aplicación de una solución normativa: de un lado porque, por vía legislativa o jurisprudencial, siempre pueden aparecer nuevas excepciones -vgr. la aparición de una nueva eximente o atenuante penal- y, de otro, porque los cambios en la interpretación del Derecho pueden también dar entrada, bajo el tipo de antiguas excepciones, a nuevas circunstancias -vgr. considerar que la inmunidad parlamentaria que exceptúa o condiciona el ejercicio de la acción penal se extiende a los representantes de una Cámara autonómica-. Es más, el problema no es sólo de defecto o de no conocer todas las excepciones, sino también de exceso, esto es, de no saber a priori qué casos no contemplados en la norma habrían de recibir sin embargo un mismo tratamiento merced al método analógico. En definitiva, la cuestión de cuándo no debemos aplicar una norma, por existir alguna excepción, así como la de hasta dónde podemos aplicarla, por resultar factible el recurso a la analogía, parece bastante más compleja de lo que dan a entender las ideas del "todo o nada" o de las "razones perentorias"». «Y paradójicamente -concluye Prieto- como ha observado Alexy, la existencia de principios explica en buena medida por qué la imagen del "todo o nada" no se adecua ni a las reglas ni a los principios. Pues si se sostiene que no cabe enumerar las excepciones a los principios y, por tanto, tampoco los supuestos de aplicación, pero se sostiene, en cambio, que los principios sí pueden exceptuar a las reglas, resultará en suma que lógicamente tampoco podemos saber los casos de excepción a las reglas».

Para responder a esta crítica debemos tratar separadamente las diversas cuestiones implicadas en ella:

1) Una primera distinción que resulta importante aquí -y que Prieto parece pasar por alto- es la que diferencia entre casos genéricos y casos individuales. Los casos genéricos no son otra cosa que conjuntos de propiedades. Por ello, un sistema de reglas que contuviera una regla general excluyente que permitiera todo aquello que no está comprendido por las prohibiciones que las reglas particulares correlacionan con las descripciones de los casos genéricos que ellas mismas contienen, carecería de lagunas normativas, sería un sistema completo, o, como prefiere decir Prieto, lógicamente finito, en relación con los casos genéricos. Otra cosa es que en la descripción de los casos genéricos hubiese problemas de indeterminación semántica, de tal forma que, frente a un caso individual (o menos genérico que el descrito en la regla), surgieran dudas acerca de su pertenencia a alguno de tales casos genéricos. 
En tal sistema, aunque carente de lagunas normativas, sí se presentarían supuestos de lo que Alchourrón y Bulygin han llamado lagunas de reconocimiento (esto es, el tipo de dudas surgidas por los problemas de indeterminación semántica a que acabamos de hacer referencia). Pues bien, en Sobre principios y reglas teníamos muy en cuenta esta distinción entre casos genéricos y casos individuales e indicábamos que asumíamos «la concepción de las normas que encontramos en Normative Systems, esto es, como correlaciones entre casos genéricos (conjuntos de propiedades) y soluciones (esto es, la calificación normativa de una determinada conducta)» y también que «las indeterminaciones semánticas» que pueda presentar una disposición «no afectan a su carácter de regla». De forma que la insistencia, plenamente razonable, de Prieto en que las reglas «ofrecen siempre una textura abierta o vaguedad potencial en su significado» ni quita ni pone en relación con el problema de la configuración «cerrada» de sus condiciones de aplicación. Porque lo que las reglas configuran a nuestro juicio de forma «cerrada» son los casos genéricos que constituyen sus condiciones (genéricas) de aplicación, mientras que el problema de la textura abierta o vaguedad potencial se da en otro ámbito, en el de la subsunción de casos individuales en tales descripciones genéricas. A este ámbito pertenece el ejemplo puesto por Prieto, cuando alude a que «los cambios en la interpretación del Derecho pueden también dar entrada, bajo el tipo de antiguas excepciones, a nuevas circunstancias -vgr. considerar que la inmunidad parlamentaria [...] se extiende a los representantes de una cámara autonómica». En tal caso, estaríamos ante un supuesto de vaguedad del término «parlamentario» que plantea dudas, finalmente resueltas positivamente, acerca de si los miembros de una asamblea legislativa autonómica están comprendidos o no en la descripción del caso genérico («ser parlamentario») de la regla que concede la inmunidad a quienes reúnan tal propiedad. Pero esto no tiene nada que ver con que la regla correspondiente configurara de forma «cerrada» sus condiciones (genéricas) de aplicación, cosa que desde luego hacía: si alguien es parlamentario, entonces goza de inmunidad; si no lo es, no goza de ella².

${ }^{2}$ La idea de que en las reglas las condiciones de aplicación están configuradas en forma cerrada (a diferencia de lo que ocurre en los principios) puede aclararse, un tanto paradójicamente, recurriendo a la obra de autores que, como Alchourrón y Bulygin, no ven precisamente con simpatía la distinción reglas / principios. En un artículo de 1988 («Condicionalidad y la representación de las normas jurídicas» en Alchourrón-Bulygin: Análisis lógico y Derecho, CEC, Madrid, 1991), Alchourrón se plantea el problema de cómo formalizar adecuadamente (esto es, en forma que sea concorde con la interpretación intuitiva común) las normas 
2) La consideración de que las reglas configuran de forma «cerrada» sus condiciones de aplicación no niega, pues, sino que es compatible con la admisión de que la descripción de tales condiciones puede presentar una zona de penumbra en relación

jurídicas. El problema consiste en que las normas subordinan el establecimiento de obligaciones o el otorgamiento de derechos a que se cumplan determinadas condiciones. Sin embargo, usualmente las normas no mencionan expresamente todas las condiciones requeridas para que surja la obligación o el derecho correspondiente, sino que, en general, suelen omitirse aquellas condiciones negativas cuya presencia impide el nacimiento de un derecho u obligación. Y ello plantea problemas del siguiente tipo. Supongamos que un sistema normativo incluye estas dos normas:

(1) Los jueces deben castigar a los que han cometido homicidio.

(2) Los jueces no deben castigar a los menores de edad.

$\mathrm{Si}$ partimos de una lógica proposicional bivalente y una lógica deóntica estándar, sus esquemas lógicos serían, respectivamente:

(3) $\mathrm{p}>$ Oq.

(4) $\mathrm{r}>\mathrm{O}$-q.

Pero de lo anterior se deriva que en el caso en que se den conjuntamente la condición de aplicación de la primera norma (haber cometido homicidio: $p$ ) y de la segunda (ser menor de edad: r), los jueces se encuentran ante un conflicto de obligaciones. Pues (en virtud de la ley de refuerzo del antecedente), a partir de (3) podría derivarse p.r >Oq, y a partir de (4), p.r >O-q. Es decir, que el sistema jurídico le lleva al juez a concluir tanto la obligación de castigar como la obligación de no castigar a quien haya cometido homicidio y sea menor de edad.

Para resolver ese problema, Alchourrón, por un lado, distingue entre el lenguaje de las normas del sistema y el metalenguaje que describe las obligaciones resultantes conforme al sistema en cuestión y, por otro lado, introduce una relación que confiere prioridad y preferencia en ciertas condiciones a una norma respecto de otra. Esto es, él entiende que en el caso en cuestión se plantea, en efecto, un conflicto de obligaciones -en el nivel de las normas-, pero el conflicto se resuelve -en el nivel del metalenguaje- porque la norma (4) tiene prioridad sobre la (3). Por ello, frente a la pregunta de «¿qué debe hacer el juez en el caso p.r.?», la respuesta es, simplemente, que no debe castigar.

Ahora bien, Alchourrón muestra que en los sistemas jurídicos que incluyen el tipo de relación ordenadora antes señalada (una relación transitiva y asimétrica) no pueden producirse conflictos de obligaciones bajo ninguna condición (salvo la imposible); pues lo obligatorio viene determinado no sólo por el contenido conceptual de las normas sino también pro su orden jerárquico. Pero lo que él parece descartar es que en un sistema jurídico existan normas que establezcan soluciones normativas incompatibles sin que se dé entre ellas la relación de orden antes indicada y sin que se trate tampoco de un caso de antinomia (por ausencia de tal relación de orden). Y esto es lo que ocurre, a nuestro juicio, con los principios: éstos establecen obligaciones configurando sus condiciones de aplicación de la manera que hemos llamado abierta, esto es, negativa -«obligatorio p salvo que esta obligación sea desplazada por un principio que en relación con el caso tenga un mayor peso»- y carente de ordenación -pues el sistema no predetermina el orden jerárquico (el «peso relativo») en caso de concurrencia de principios. De ahí que para resolver un caso en el que están involucrados principios sea precisa una operación intermedia, esto es, el estable- 
con la cual sea dudosa la subsunción de un determinado caso individual. Tampoco nos parece, desde luego, que plantee dificultades a nuestra concepción el hecho de que "por vía legislativa o jurisprudencial, siempre pueden aparecer nuevas excepciones» a la aplicabilidad de una regla. Que las autoridades normativas del sistema pueden cambiar éste, restringiendo las condiciones de aplicación de una determinada solución normativa es, naturalmente, algo obvio; la consideración de que las reglas configuran de forma «cerrada» sus condiciones de aplicación se limita, como es fácil suponer, a las reglas existentes en un momento dado y no implica, desde luego, que quienes la sostenemos nos atribuyamos ninguna forma de presciencia de la conducta futura de las autoridades normativas. El conocimiento por parte de Dios de los futuros contingentes ya plantea suficientes y arduos problemas a los teólogos como para que nadie desee compartirlo.

3) La admisibilidad de solucionar normativamente un caso no regulado por el sistema mediante el argumento por analogía, aunque es un problema más serio, tampoco nos parece que plantee especiales dificultades a nuestra concepción. Lo que la admisibilidad del argumento por analogía muestra es precisamente que, ante la imposibilidad de subsumir un caso individual dentro de las condiciones de aplicación de una regla preexistente (y precisamente porque estas condiciones de aplicación tienen carácter «cerrado»), el juez ha de construir, para que sirva de fundamento a su decisión, una regla general que correlacione otras condiciones de aplicación con la misma solución normativa que una regla preexistente correlaciona con condiciones de aplicación que el juez entiende como sustancialmente semejantes. Y tal relación de semejanza no puede afirmarse (o negarse) más que en base al principio que explica y justifica la regla preexistente. Es decir, el argumento por analogía implica siempre una utilización de principios, aunque ello no signifique tampoco que el recurso a la analogía y a los principios sea una misma cosa. En realidad, la analogía es un argumento -o, mejor, una estructura de argumentación-, mientras que los principios son un material que necesariamente debe usarse en ese tipo de argumentación.

4) Prieto no objeta nuestra tesis de que los principios configuran de forma «abierta» sus condiciones de aplicación. Pero afirma que de ello se sigue una consecuencia que invalidaría

cimiento (a partir de dichos principios) de una nueva regla. A esa operación consistente en transformar los principios en reglas es a lo que se suele llamar concreción. Sobre ello volveremos más adelante, a propósito de lo que llamábamos la fuerza expansiva de los principios y que, según nos ha hecho notar Peczenik, no explicábamos suficientemente. 
nuestra caracterización de las reglas: «si se sostiene -escribe- que no cabe enumerar las excepciones a los principios y, por tanto, tampoco los supuestos de aplicación, pero se sostiene, en cambio, que los principios sí pueden exceptuar a las reglas, resultará en suma que lógicamente tampoco podemos saber los casos de excepción a las reglas». En esto Prieto tiene razón, pero ello no tiene para nuestra propuesta las consecuencias devastadoras que él presume, aunque desde luego nos obliga a precisar -como ya lo hicimos en Sobre principios y reglas- los límites dentro de los cuales asumimos la tesis de que las reglas configuran de forma «cerrada» sus condiciones de aplicación y constituyen -cuando se dan tales condiciones de aplicación- razones perentorias.

Es cierto, a nuestro juicio, que la aplicabilidad de toda regla está condicionada a que no suceda que su aplicación entre en conflicto con un principio que, en relación con las propiedades relevantes del caso, tenga un mayor peso. Tras reconocer esto uno puede, desde luego, concluir, al modo de los realistas, que las reglas no son más que juguetes vistosos, que el razonamiento jurídico es siempre radicalmente abierto, y entregarse, a continuación, al gimoteo sobre la indeterminación tan habitual en los autores de Critical Legal Studies. El problema de tal tipo de orientaciones es -como tantas veces se ha puesto de relieve- que chocan con la evidencia de que, en relación con la inmensa mayoría de los casos individuales que se presentan ante los tribunales, su subsunción bajo el caso genérico contemplado en una regla general -y el dictado de una sentencia así fundamentada- no da lugar a ningún tipo de controversia entre la comunidad jurídica.

Para poder dar cuenta adecuadamente de ambas circunstancias -la subordinación de la aplicabilidad de las reglas a los principios y el hecho de que la inmensa mayoría de los casos se solucionan mediante la aplicación no controvertida de reglas- propusimos entender la manera como el Derecho guía el razonamiento de sus órganos de aplicación como una estructura de dos niveles: en un primer nivel, decíamos, el Derecho «impone a los órganos jurisdiccionales el deber de componer un balance de razones integrado únicamente por las constituidas por las pautas jurídicas, siendo admisible la toma en consideración de otras razones únicamente en la medida en que las propias pautas jurídicas lo permitan»; en un segundo nivel, «tal balance de razones remite, no en todos los casos pero sí en la mayoría, a adoptar como base de la resolución una regla jurídica, esto es, una razón perentoria». De modo que la configuración «cerrada» por parte de las reglas de sus condiciones de aplicación (y la operatividad de las propias reglas como razones 
perentorias) sólo opera, a nuestro juicio, en este segundo nivel. Al que se pasa, por cierto, cuando el principio de que «debe hacerse lo prescrito por las reglas jurídicas» no resulta desplazado, en el primer nivel, por un principio que, en relación con el caso, tenga un mayor peso. Pero cuando tal principio de que «debe hacerse lo prescrito por las reglas jurídicas» no resulta desplazado, la regla jurídica bajo la que se subsume el caso individual opera en el razonamiento del órgano jurisdiccional como una razón perentoria para dictar una resolución cuyo contenido corresponda al de la regla.

\section{a”) Las críticas de Peczenik}

Aleksander Peczenik impugna nuestra consideración de que los principios constituyen meramente razones de primer orden para decidir en un determinado sentido mientras que las reglas serían razones «perentorias» o «protegidas». Escribe, así, que

«no sólo los principios, sino también algunas reglas crean un deber meramente prima-facie y por consiguiente exigen deliberación».

Y más adelante:

«Me parece que las “pautas jurídicas” permiten tomar en cuenta todas las consideraciones moralmente relevantes [...]. Las normas jurídicas establecidas constituyen razones prima-facie, que han de ser sopesadas y ponderadas con otras razones. Estas razones prima-facie son razones de primer orden para la realización de una cierta acción, H, y, al mismo tiempo, razones de segundo orden. En esta última capacidad, indican que las razones para no hacer $\mathrm{H}$ pueden prevalecer sólo si son particularmente fuertes, esto es, claramente más fuertes de lo que necesitan ser en un debate moral libre».

Estas tesis de Peczenik -meramente enunciadas en el trabajo al que respondemosse encuentran considerablemente desarrolladas en su importante libro On Law and Reason (Kluwer, Dordrecht / Boston/ London, 1989), al que él mismo remite. Dentro de él, en la sección titulada Weighing Rules, leemos lo siguiente:

«Sin embargo, no sólo los principios, sino también algunas reglas crean un deber meramente prima-facie. Esto es verdadero tanto de las reglas morales como de las reglas jurídicas. Por ejemplo, no se debe matar a las personas. La regla moral prohíbe 
prima-facie todo acto de matar, pero para afirmar que un determinado individuo, consideradas todas las cosas, debe no ser matado, se debe prestar atención también a otras reglas que estipulan excepciones; por ejemplo, en una guerra defensiva, se puede matar a los agresores [...]. Todas las normas jurídicas socialmente establecidas, expresadas en disposiciones legislativas, precedentes, etc., tienen un carácter meramente prima-facie. El paso de las reglas jurídicas prima-facie a las obligaciones, pretensiones, etc., jurídicas (y morales) implica interpretación valorativa, esto es, sopesar y ponderar» (pp. 80-1).

A nuestro modo de ver, a este planteamiento de Peczenik cabe oponer un par de consideraciones:

1) La primera es que el ejemplo de regla que crea un deber meramente prima-facie (la prohibición de matar) no sería tal, de acuerdo con nuestras definiciones, sino un principio. Y ello porque dicha norma no determina sus condiciones de aplicación más que de la forma que nosotros llamamos abierta (y a la que consideramos característica de los principios): «está prohibido matar a menos que concurra otro principio que, en relación con el caso, tenga un mayor peso». Puede objetarse, desde luego, trazar una distinción conceptual entre tipos de normas jurídicas, como la que nosotros trazamos entre reglas y principios, atendiendo al carácter «cerrado» o «abierto» de la determinación de las condiciones de aplicación: sosteniendo, por ejemplo, que tal rasgo no es particularmente relevante, que no refleja el uso lingüístico habitual de los términos "regla" y "principio", etc. Pero si nuestra distinción así trazada no se objeta, es claro que el «no matarás» no es (a diferencia, por cierto, de las reglas sobre el homicidio contenidas en el Código Penal) una regla sino un principio. Resulta, además, que de acuerdo con la propia caracterización que Peczenik ofrece de la distinción entre reglas y principios, habría que concluir que la prohibición de matar debe entenderse asimismo como un principio. Así parecen indicarlo sus observaciones de que «a diferencia del principio, la regla [...] no expresa un valor singular sino un compromiso entre muchos valores (y los correspondientes principios)» (On Law and Reason, p. 81), y de que «la principal fuente de la fuerza justificatoria de los principios consiste en su vínculo uno-a-uno con los correspondientes valores. Cada principio responde a un valor determinado [...]» (Los principios jurídicos según M. Atienza y J. Ruiz Manero).

2) La segunda consideración se refiere a la tesis de Peczenik de que las pautas jurídicas permiten tomar en cuenta todas las 
consideraciones moralmente relevantes, de que las normas jurídicas establecidas constituyen razones prima-facie, que en su dimensión de razones de segundo orden se limitan a indicar que las razones para no realizar la acción exigida por ellas sólo pueden prevalecer si son particularmente fuertes. Esta tesis es, a nuestro juicio, ambigua. Pues puede entenderse (i) como una tesis que pretende dar cuenta de las pretensiones del Derecho o (ii) como una tesis que pretende dar cuenta de la actitud que un sujeto práctico-racional ha de adoptar frente a tales pretensiones. En ninguno de ambos casos resulta, a nuestro modo de ver, aceptable. Entendida como referida a las pretensiones del Derecho, la tesis resulta ser claramente falsa. Por poner un ejemplo en el que es difícil que se produzca controversia: imponer a alguien una restricción en sus derechos básicos por razón de su raza es contrario a la consideración, moralmente relevante, de que tales derechos básicos deben adscribirse a todos los seres humanos, por el simple hecho de serlo. Y es obvio que ha habido ordenamientos que no permitían tomar en cuenta tal consideración. Si la tesis de Peczenik se entiende, por el contrario, como referida a la actitud que un sujeto práctico-racional ha de adoptar frente a las pretensiones del Derecho, dicha tesis concede, a nuestro juicio, demasiado. Decir que un sujeto práctico-racional ha de considerar siempre a las normas jurídicas establecidas como razones prima-facie para realizar la conducta prescrita por ellas equivale a decir que un sujeto práctico-racional ha de aceptar que existe una obligación moral general prima-facie de obedecer al Derecho. Esto es algo en lo que Aleksander Peczenik ha insistido repetidas veces (cf. On Law and Reason, pp. 238 ss.; «Dimensiones morales del Derecho», en Doxa, n. ${ }^{\circ}$ 8, pp. 96 ss.), pero su argumentación no resulta, a nuestro juicio, convincente. «El punto central», como él mismo dice, de su teoría es el siguiente: «Existe una obligación moral general prima-facie de obedecer el Derecho porque la desobediencia general crearía el caos» (On Law and Reason, p. 246). La justificación de esta tesis por parte de Peczenik no apela a la incidencia causal que los actos de desobediencia tengan sobre la conservación del sistema, sino al requisito de universalización. Peczenik acepta que hay actos de desobediencia que no incrementan en modo alguno la probabilidad de otros actos de desobediencia y considera correcta la crítica de Raz a los intentos de fundamentar sobre la base de la incidencia causal (del «mal ejemplo») la tesis de la existencia de una obligación moral general prima-facie de obedecer al Derecho. Peczenik fundamenta su tesis sobre la base de la siguiente «premisa universal», que «es una consecuencia del carácter universalizable de la moral»: "Yo tengo una obligación moral prima-facie de 
actuar de tal manera que mi acción pudiera repetirse por todos sin crear consecuencias moralmente incorrectas» (On Law and Reason, p. 246). Pero si el principio con arreglo al cual actúo yo es el de que «es moralmente legítimo desobedecer cuando mi acto de desobediencia no va a incrementar en modo alguno la posibilidad de otros actos de desobediencia y no va, por consiguiente, a afectar causalmente a la conservación del sistema» es analíticamente verdadero que cualquiera puede actuar sobre la base de tal principio (esto es, que tal principio puede universalizarse) sin que de ello se sigan consecuencias moralmente incorrectas (el incremento de probabilidad de otros actos de desobediencia y, como consecuencia, el derrumbe del sistema) ( $c f$., sobre este punto, Juan Carlos Bayón: La normatividad del Derecho. Deber jurídico y razones para la acción, CEC, Madrid, 1991, pp. 708-9). Realmente, el argumento de Peczenik no sirve de justificación de su tesis, como tampoco serviría como justificación del juicio de que «existe una obligación general prima-facie de no circular por el punto kilométrico $\mathrm{X}$ de la carretera $\mathrm{Z}$ a la hora $\mathrm{H} »$, aducir que si todo el mundo circulara por el punto kilométrico $\mathrm{X}$ de la carretera $\mathrm{Z}$ a la hora $\mathrm{H}$ se crearía un colapso circulatorio. Pero todo esto nos sitúa más allá de los límites de nuestro trabajo sobre los principios en el Derecho.

\section{b) Principios y cumplimiento pleno}

Con posturas asimismo diferentes entre sí, Prieto y Peczenik concuerdan en rechazar nuestra tesis de que los principios en sentido estricto no admiten, a diferencia de las directrices, modalidades graduales de cumplimiento.

b') La postura de Prieto.

Bajo el epígrafe de Caracterización normativa de los principios, se opone Prieto a la consideración, deudora de algunas sugerencias de Dworkin, de que los principios no constituyen nunca razones suficientes para las decisiones, de que éstos «pueden orientar una interpretación normativa dudosa, pero nunca pueden por sí solos ofrecer la solución al caso», pues «ni ofrecen ni dejan de ofrecer una respuesta categórica, sino que "controlan" (ampliando o limitando) las soluciones que se deducen del conjunto de las normas» ${ }^{3}$. «Pienso -escribe Prieto- que ésta es una interpretación

${ }^{3}$ Entre quienes han sostenido esta visión de los principios cita Prieto su 
aceptable de las palabras de Dworkin, pero probablemente constituya también una opinión equivocada». Para argumentar lo equivocado de esta opinión, apela Prieto a la jurisprudencia de nuestro Tribunal Constitucional y a los propios ejemplos de Dworkin. Por lo que hace a lo primero, recuerda que el TC ha declarado que, cuando la oposición entre las leyes y los principios (constitucionales) sea irreductible, éstos participan de la fuerza derogatoria de la Constitución, lo que implica que «al menos en ciertos supuestos un principio representa el fundamento único de la decisión», lo que se produce cuando el TC declara la inconstitucionalidad de una disposición legislativa porque la misma vulnera un principio constitucional. Y por lo que hace al famoso ejemplo dworkiniano que Carrió bautizara como «el caso del nieto apurado», escribe Prieto que «si, de acuerdo con las leyes de New York los descendientes suceden a los ascendientes y ninguna regla excepciona el caso del nieto que asesina a su abuelo, ello significa que si un tribunal impide tal consecuencia en virtud del principio nemine dolus suus prodesse debet, dicho principio representa el fundamento único de la decisión; no cabe decir que el principio ha sido tenido en cuenta para inclinarnos en favor de una u otra solución normativa, avaladas ambas por reglas, pues aquí sólo existen dos soluciones: aquélla (única) que se debería adoptar de observarse las reglas, y aquella otra que imponen los principios».

Hasta aquí estamos por completo de acuerdo con Prieto. Y nos parece que lo que muestran sus dos ejemplos es que, una vez que se ha determinado la prevalencia de un determinado principio en un caso, el principio en cuestión exige un cumplimiento pleno, lo que implica, en los ejemplos mencionados, la anulación de la ley inconstitucional y el rechazo de la pretensión del nieto de entrar en posesión de la herencia de su abuelo. Sin embargo, pocas páginas más tarde, Prieto hace suya lo que podríamos llamar una versión debilitada de la idea de Alexy del «mandato de optimización», defendiendo no sólo la idea de las modalidades graduables de cumplimiento, sino que lo que los principios exigen es meramente un grado de cumplimiento «razonable»:

[...] la idea del mandato de optimización [...] tampoco me parece que sirva para diferenciar los principios en sentido estricto de las directrices, como mantienen Atienza y Ruiz Manero, pues los principios, cuando existe conflicto, también pueden ser optimizados;

propio trabajo «Teoría del Derecho y filosofía política en Ronald Dworkin» (en Revista Española de Derecho Constitucional, 14, 1985) y el de J. Ruiz Manero Jurisdicción y Normas, CEC, Madrid, 1990. 
acaso cabría decir que las directrices generan naturalmente mandatos de optimización, pero sin que ello implique que esta técnica quede totalmente excluida de los principios en sentido estricto. Trataré de explicarlo con un ejemplo: imaginemos una cláusula testamentaria en la que un padre impone a su hijo como condición para acceder a los bienes (legítima y libre disposición) que se divorcie de su mujer, de religión judía. Para resolver el caso, el juez debe tener presente dos principios: el de igualdad, que prohíbe la discriminación religiosa y racial, y el de autonomía de la voluntad, que protege las intenciones del testador. Pues bien, no sería del todo extraño que aquí se renuncie sin más a la idea de optimización, dando total preferencia al principio de igualdad o -lo que me parecería peor solución- al de autonomía de la voluntad; pero tampoco sería sorprendente que algún juez intente ponderar ambos principios, diciendo que el testamento es nulo por lo que se refiere a la legítima, pero válido en relación con los bienes de libre disposición.

Sin duda, es cierto que el cumplimiento gradual de algunos estándares y el juicio de optimización son ideas presentes en el razonamiento jurídico, pero tal vez resulte apresurado decir que los principios ordenan siempre al intérprete que se realice algo en la mayor medida posible. Como ha declarado el Tribunal Constitucional, "la Constitución es un marco coincidencias suficientemente amplio como para que dentro de él quepan opciones políticas de muy diferente signo", y sin duda, los programas de esas opciones pueden presentar ideas distintas sobre el grado de realización de los principios o directrices constitucionales. Por ello, desde la perspectiva del juez, más que de un juicio de optimización habría que hablar de un juicio de razonabilidad, que, aceptando la idea de cumplimiento gradual, indique por debajo de qué nivel de cumplimiento o satisfacción una determinada norma o política se hace intolerable».

Esta última idea se encuentra más desarrollada por el propio Prieto en su trabajo «Notas sobre la interpretación constitucional» (en Revista del Centro de Estudios Constitucionales, 9, 1991, pp. 175 ss.). En dicho trabajo, bajo el rótulo de «algunas peculiaridades de la justicia constitucional», escribe Prieto lo que sigue:

«La diferencia entre la interpretación legal y la constitucional no reside sólo en las peculiaridades de su objeto, sino también en la función que generalmente se atribuye a los órganos encargados de realizarla. En este aspecto, una de las características del juez ordinario es lo que pudiera llamarse "unidad de solución justa", esto es, la exigencia institucional de que, en presencia de un caso concreto, sólo cabe una interpretación correcta, mientras que, en cambio, la misión de la justicia constitucional no es tanto la de 
precisar la "mejor" o la "única" respuesta posible, sino más bien la de indicar qué interpretaciones resultan intolerables [...]. Esta distinta función [...] contribuye también a entender de modo diferente la responsabilidad de la decisión. Así, y aunque se trate sin duda de una ficción, el juez ordinario puede "endosar" el sentido del fallo al propio legislador [...]. En cambio, el intérprete constitucional no busca en realidad una solución al caso, sino la delimitación de un campo de licitud dentro del cual otros operadores jurídicos adoptarán la solución con arreglo a criterios políticos (legislador) o jurídicos (juez); por eso, su modo de argumentar no puede ajustarse a los cánones de la subsunción, sino a los de la razonabilidad [...]. Dicho de otro modo, el tipo de razonamiento de un juez ordinario supone concebir la decisión "como si" derivase del legislador, mientras que el modelo de razonamiento del juez constitucional, al tener que definir el ámbito más o menos extenso de licitud, reclama del intérprete la asunción de una mayor responsabilidad en la decisión. En este sentido, creo que tiene razón A. Carrasco cuando escribe que el método característico de la interpretación constitucional se sitúa a medio camino entre lo que él llama la deducción estricta propia de la justicia ordinaria y el juicio de optimización política; de un lado, y por la misma naturaleza de su actuación, el Tribunal Constitucional no está en condiciones de verificar una mera labor de subsunción, pues generalmente ni aquello que ha de ser enjuiciado se asemeja a un supuesto de hecho, ni los parámetros para el enjuiciamiento pueden dejar de ponderar conjuntamente principios y reglas; $y$, de otra parte, ha de "autocontenerse" también a fin de no realizar un juicio de optimización que implicaría decidir cuál es la "mejor" interpretación del texto constitucional y, consiguientemente, asfixiar el margen de apreciación del Parlamento» (op. cit., pp. 176-8).

A nuestro juicio, lo que falla en todas estas consideraciones respecto al entendimiento de la jurisdicción constitucional es algo que se conecta con la manera, en nuestra opinión incorrecta, como Prieto entiende el papel de los principios en el razonamiento de los órganos jurisdiccionales. Dicho rápidamente, el error reside básicamente en presentar como características peculiares de la justicia constitucional lo que son rasgos propios de cualquier ejercicio de la función jurisdiccional en la que lo enjuiciado sean actos que constituyan un ejercicio de poderes normativos en los que el titular del poder goza de un margen de discrecionalidad. Veámoslo. Dice Prieto que «la misión de la justicia constitucional no es tanto la de precisar la "mejor" o la "única" respuesta posible, sino más bien la de indicar qué interpretaciones resultan intolerables [...]. El intérprete constitucional no busca en realidad una solución al caso, sino la delimitación de un campo de licitud 
[...]; por eso, su modo de argumentar no puede ajustarse a los cánones de la subsunción, sino a los de la razonabilidad». Desde luego que la justicia constitucional no tiene como misión precisar cuál es, desde la perspectiva constitucional, la mejor ley posible; pero tampoco es misión de un juez de lo contencioso precisar cuál es el mejor reglamento posible desde la perspectiva de la ley que ese reglamento desarrolla, ni tampoco es misión de un juez de lo civil precisar cuál es el mejor contrato o el mejor testamento posible desde la perspectiva del Código civil. Tales cosas sencillamente no existen: ni a partir de la Constitución es posible derivar la «mejor» legislación o la «única» legislación admisible en una determinada materia, ni a partir del Código civil es posible derivar el «mejor» contrato o testamento o el «único» admisible. Lo que hacen la Constitución y el Código civil es conferir, respectivamente, el poder normativo público de legislar y los poderes normativos privados de celebrar contratos $u$ otorgar testamento, imponiendo a la vez determinadas restricciones al ejercicio de esos poderes normativos. Y lo que hace el Tribunal Constitucional cuando enjuicia la constitucionalidad de una ley, o un juez de lo civil cuando enjuicia la validez de un contrato o de un testamento, es enjuiciar si los titulares de los poderes normativos correspondientes han usado éstos de forma que viole las referidas restricciones o no. Y respecto de ello, tanto el Tribunal Constitucional como el juez de lo civil han de buscar -en contra de lo que parece pensar Prieto en el primer supuesto- una solución al caso: si el legislador ha usado su poder normativo público violando las restricciones impuestas por la Constitución, el Tribunal Constitucional debe declarar la inconstitucionalidad de la ley en todo lo que abarque dicha violación; si el testador ha usado su poder normativo privado violando las restricciones impuestas por el Código civil, el juez debe declarar la invalidez del testamento en todo lo que abarque dicha violación. Y si uno y otro han ejercido sus respectivos poderes normativos de forma que no viole las referidas restricciones, el Tribunal Constitucional y el juez de lo civil han de declarar la constitucionalidad de la ley y la validez del testamento, por extravagantes que puedan parecerles las disposiciones de una $\mathrm{u}$ otro: del mismo modo que el Tribunal Constitucional -como escribe Prieto- ha de «autocontenerse a fin de no realizar un juicio de optimización» respecto de cuál sería la mejor ley posible, también el juez de lo civil ha de «autocontenerse» a fin de no realizar un juicio de optimización respecto de cuál sería el mejor testamento posible, o el más razonable o cosas por el estilo. Y uno y otro -Tribunal Constitucional y juez de lo civil- han de fundamentar su resolución del mismo modo: mostrando que la 
ley (o el testamento) han violado (o no) las restricciones impuestas por la Constitución (o por el Código civil). En este orden de cosas, la vinculación del Tribunal Constitucional a la Constitución y la del juez ordinario a la ley no presentan ninguna diferencia relevante. Y tanto uno como otro pueden enfrentarse a un caso en el que hayan de tener en cuenta tanto reglas como principios, como muestra el propio ejemplo de Prieto respecto al testamento sujeto a la condición de divorcio de la esposa judía. Ejemplo sobre el que, por cierto, vale la pena detenerse brevemente. De acuerdo con la reconstrucción de Prieto, el juez podría adoptar respecto de él tres soluciones: considerar nulo todo el testamento, lo que significaría dar «total preferencia al principio de igualdad»; considerar válido todo el testamento, lo que significaría dar «total preferencia al principio de igualdad»; considerar válido todo el testamento, lo que significaría dar total preferencia al principio de autonomía de la voluntad; considerar nulo el testamento por lo que se refiere a la legítima y válido por lo que hace a los bienes de libre disposición, lo que significaría que el juez ha intentado «ponderar ambos principios». Esta reconstrucción es, a nuestro juicio, equivocada. Las cosas se plantean más bien, a nuestro modo de ver, de la manera siguiente. La concurrencia entre el principio de autonomía de la voluntad y el principio de igualdad se produce únicamente en relación con los bienes de libre disposición. En relación con la legítima, el caso se encuentra cubierto por la regla de que los descendientes tan sólo pueden ser privados de tales bienes por causas tasadas, entre las que, desde luego, no se encuentra el estar casado con persona de religión judía. Tal regla puede verse como expresión de la prevalencia, establecida por el legislador en relación con una parte del caudal hereditario, del principio de protección de los intereses de los descendientes sobre el principio de respeto a la autonomía de la voluntad del testador. $\mathrm{Y}$, salvo que se argumente (lo que no hace Prieto) que la jerarquización entre principios expresada en la regla sobre la legítima choca, en el caso en cuestión, con algún otro principio, el caso es, por lo que se refiere a la legítima, fácil, al no resultar controvertible la aplicación de una regla cuyas condiciones genéricas de aplicación cubren por completo las circunstancias especificas del caso. Las cosas se plantean de manera distinta por lo que se refiere a los bienes de libre disposición. Y ello porque, a diferencia de lo que ocurre con la legítima, aquí no disponemos de una regla que establezca la prevalencia entre el principio de autonomía de la voluntad y algún otro principio eventualmente concurrente (en el caso en cuestión, el principio de igualdad). Y por ello el juez debe ponderar ambos principios, esto es, construir 
una regla que establezca dicha prevalencia. Y aquí caben dos y sólo dos posibilidades: o prevalece el principio de autonomía de la voluntad (y las cláusulas testamentarias correspondientes son, por consiguientemente, válidas) o prevalece el principio de igualdad (y dichas cláusulas son nulas). En otros términos: una vez establecida la prevalencia de uno u otro principio, éste exige un cumplimiento pleno: la declaración de validez o de nulidad de las cláusulas testamentarias correspondientes. Tertium non datur.

\section{b”) La postura de Peczenik}

Peczenik ofrece dos argumentos en defensa de la concepción alexiana de los principios como mandatos de optimización y en contra de nuestra tesis de que los principios en sentido estricto exigen un cumplimiento pleno. El primero de los argumentos de Peczenik es que el ejemplo de principio en sentido estricto con el que ilustrábamos nuestra tesis (el art. $14 \mathrm{CE}$ ) está mal escogido, pues tal disposición no expresaría un principio en el sentido de Alexy:

«el ejemplo no falsa la teoría de Alexy, dado que el art. 14 de la Constitución española es una regla (vaga), no un principio en el sentido de Alexy. Por otra parte, disposiciones tales como el Cap. 1 Sec. 2 de la Constitución sueca, que son principios en este sentido, pueden ser cumplidos en diversos grados»».

Realmente, no resulta fácil responder a este argumento. Y ello, en primer lugar, porque Peczenik no nos explica por qué el art. $14 \mathrm{CE}$ no es un principio en el sentido de Alexy y qué diferencias relevantes hay, a este respecto, entre el art. $14 \mathrm{CE}$ y la disposición de la Constitución sueca por él citada. Dicha disposición establece que «el poder público se debe ejercer con respeto al igual valor de todos los seres humanos y a la libertad y dignidad de cada persona individual». ¿Por qué habría que concordar con Peczenik en que tal disposición expresa tres principios -igualdad, libertad y dignidad de cada persona individual- y negar, en cambio, que el art. $14 \mathrm{CE}$ exprese el principio de igualdad? Tanto en la disposición citada de la Constitución sueca como en el art. 14 CE se da el «vínculo uno-a-uno» con los correspondientes valores que Peczenik considera característico de los principios. Ni una ni otra disposición determinan sus condiciones genéricas de aplicación. ¿Cuál es la diferencia entre ambas que permite sostener que la primera expresa tres principios y la segunda ninguno? Por 
los demás, Peczenik afirma que los principios contenidos en la Constitución sueca pueden ser cumplidos en diversos grados. Pero su afirmación equivale a dar por supuesto precisamente lo que habría de argumentar, esto es, la posibilidad de modalidades graduables de cumplimiento de cada uno de estos tres principios.

El segundo argumento ofrecido por Peczenik en defensa de la concepción de Alexy se encuentra en el siguiente párrafo, en el que, tras citar nuestra afirmación de que los principios tienen una «fuerza expansiva» superior a la de las reglas, escribe:

«Sin intentar explicar el término "fuerza expansiva", se puede indicar que la principal fuente de la fuerza justificatoria de los principios consiste en su vínculo uno-a-uno con los correspondientes valores. Cada principio responde a un valor determinado, por ejemplo él estipula que la igualdad, la libertad y la dignidad son valiosas... [...] Un valor puede ser definido como un criterio de valoración. Cada criterio puede satisfacerse hasta un cierto grado, mayor o menor [...]. Cada principio exige que el valor al que él mismo corresponde sea respetado tanto como sea posible. Pero si éste es el caso, la posibilidad de cumplir los principios en diversos grados, mayores o menores, es la propiedad más esencial de los principios. Y Alexy tiene razón mientras que la crítica de su teoría por parte de Atienza y Manero no es suficientemente profunda».

Estamos de acuerdo con Peczenik en que es característico de los principios en sentido estricto lo que él llama su «vínculo uno-a-uno con los correspondientes valores». De hecho, en nuestro trabajo caracterizábamos los principios en sentido estricto en términos muy semejantes. Pero de ahí no se infiere en absoluto lo que Peczenik pretende inferir. Ciertamente que los principios constituyen criterios de valoración de conductas. Pero es sencillamente falso decir que siempre caben modalidades graduables de satisfacción de un criterio de valoración. El mejor ejemplo en contra lo encontramos en aquellas pautas jurídicas a las que llamamos reglas, las cuales, para quien las acepta, constituyen también criterios de valoración de las acciones. Y es indisputado que las reglas no admiten modalidades graduables de cumplimiento: sencillamente se incumplen. Lo mismo ocurre, a nuestro juicio, con los principios en sentido estricto, y no llegamos a encontrar en Peczenik razones que nos induzcan a revisar nuestra posición.

Para terminar, dos palabras sobre lo que llamábamos «fuerza expansiva» de los principios y que, desde luego, apenas esbozábamos en nuestro trabajo. En relación con los principios en sentido estricto, la «fuerza expansiva» de los mismos se traduce, por 
ejemplo, en la generación de reglas en la que se determine su prevalencia (o no) en un determinado caso genérico. Volvamos al ejemplo del testamento de Prieto. Si el tribunal que ha de resolver determina la prevalencia del principio de igualdad (o del principio más concreto de prohibición de discriminación por razones de conciencia) sobre el de autonomía de la voluntad, tal determinación se traduce en la construcción, como fundamento de su decisión, de una regla que establezca que en determinadas condiciones genéricas (testar, celebrar un negocio jurídico privado) está prohibido discriminar por razones de conciencia. Y si las rationes decidendi del tribunal que resuelve el caso son vinculantes para los demás órganos jurisdiccionales, dicha regla pasa a ser una regla del sistema jurídico de que se trate. 